\title{
Analysis of the results of a survey on a residential building after a gas explosion and fire
}

\author{
Dmitry Panfilov ${ }^{1, *}$, Alexander Zaitsev ${ }^{1}$, and Evgeny Yudin ${ }^{1}$ \\ ${ }^{1}$ Voronezh state technical university, 394006, Voronezh, 20- letia oktyabrya, 84, Russia
}

\begin{abstract}
The paper analyzes the statistics on household gas explosions and subsequent fires in the residential sector. The results of a survey of a residential building after a gas explosion and a real fire are presented in order to determine the possibility of its restoration and further operation. The results of the necessary experimental and theoretical studies are presented. Examples of the use of foreign experience in the study of fire resistance of building structures are given. The results obtained were used in the development of recommendations for the possibility of restoration of the house and during the necessary construction and finishing works.
\end{abstract}

\section{Introduction}

Hundreds of natural gas explosions occur in Russia every year, in just the last two years, the total number of household gas explosions in residential apartment buildings has made up more than 61 cases. In fig. 1 some statistics on the issue under consideration are presented. Statistics show that there is a tendency to an increase in cases of destruction of houses and apartments, as well as to the death and injury of people [1]. Often the explosion of the resulting air-gas mixture leads to large-scale destruction with a significant probability of fire. Always, one of the sides of this problem is the question of the possibility of restoration and further operation of the damaged building.

\footnotetext{
${ }^{*}$ Corresponding author: panfilov_dv@vgasu.vrn.ru
} 


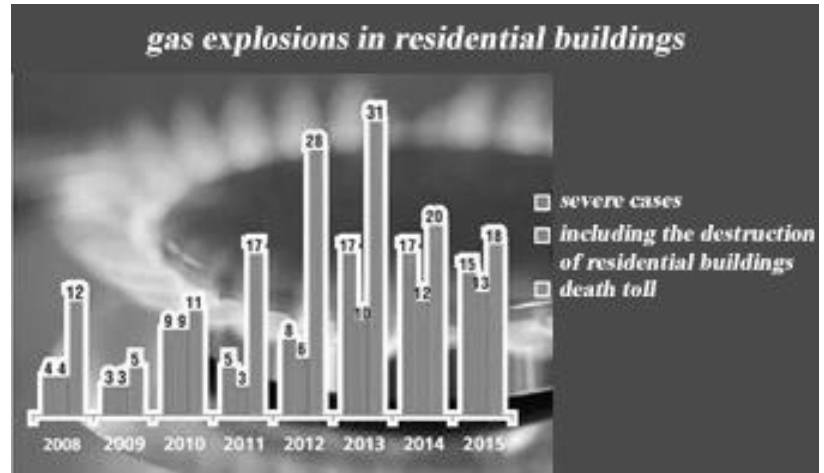

Fig. 1. Statistics of explosions and fires of domestic gas.

\section{Materials and methods}

This article presents an analysis of the results of a study on the possibility of restoring and operating a residential apartment building after an explosion and a real fire.

A gas explosion occurred in a residential building in Voronezh on Kosmonavtov street, 5, according to the Ministry of Emergencies, on January 11, 2016 at 21:30 Moscow time. The fire was preceded by a powerful explosion, then the fire embraced the third and fourth floors of a five-storey building. Rescuers quickly left the scene, evacuating 30 people from the burning building. By 10:40 p.m. Moscow time, the fire on Kosmonavtov Street was eliminated. Residents were not allowed into the house that exploded, since there was a danger of collapsing partitions. Fire severely damaged a 1966 panel building.

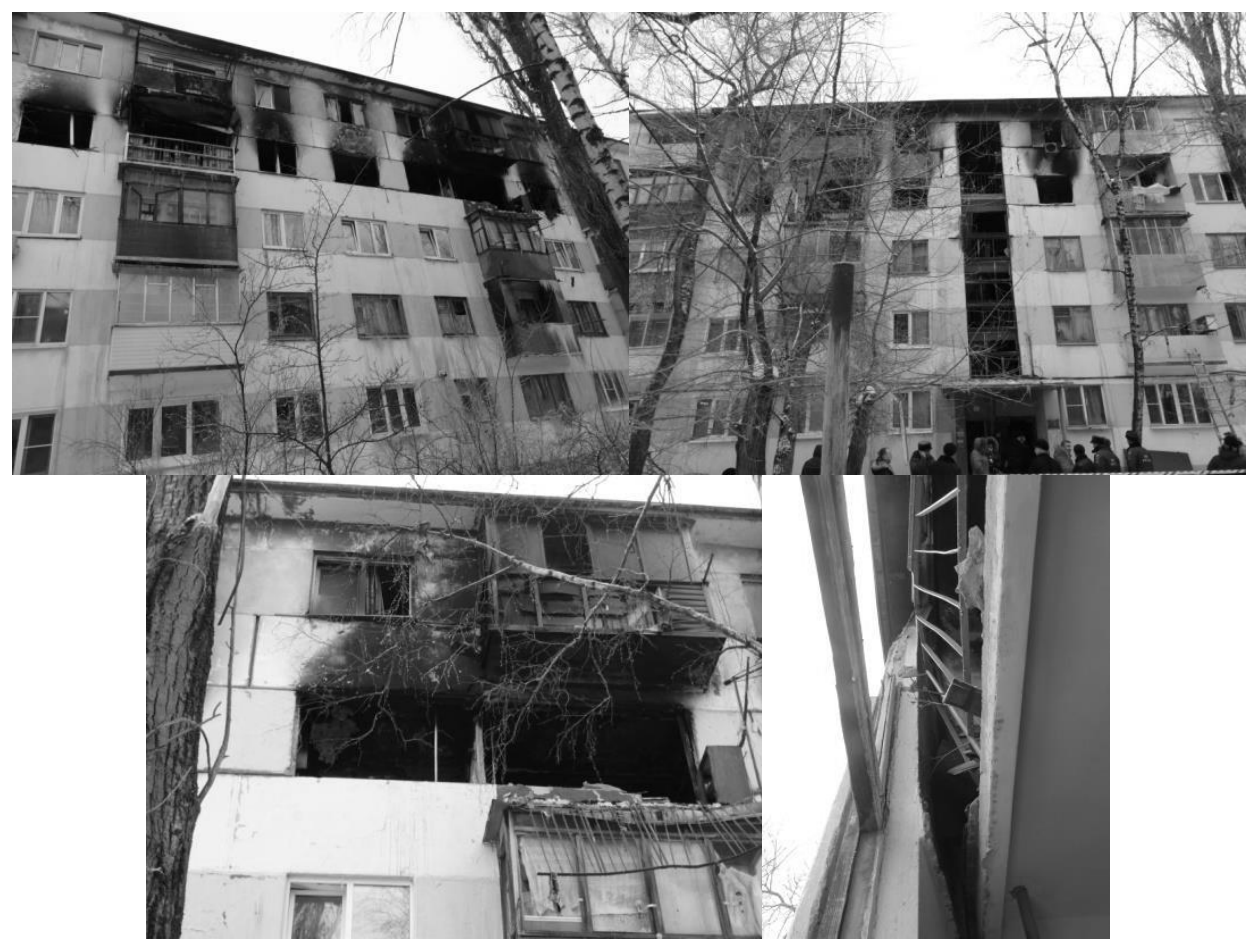

Fig. 2. Photos of the house after the explosion and fire. 
The examination was carried out in order to identify defects and damage to the main load-bearing building constructions of the 1 st to 5 th floors of the 3rd entrance of a residential building (including load-bearing reinforced concrete walls adjacent to the 2 nd and 4th entrances, including the 3rd entrance as a result of the explosion and fire.

To determine the possibility of restoration and further operation of the residential building, the following studies were carried out: instrumental and laboratory examination of the condition of load-bearing reinforced concrete structures. Applied methods for inspection of structures included the following steps:

- study of materials of operational services for the occurrence and liquidation of emergencies;

- the study of photos and videos from the scene to record the intensity and time of the thermal effect on building constructions, as well as other parameters characterizing the development and elimination of emergencies;

- visual inspection with photographing of defects and damages, including nodes and interfaces of the main structures;

- geodetic survey of fixed structures and adjacent to the collapse or destruction zone, including facades;

- determination of the parameters of materials exposed to fire;

- verification calculation of established structures and adjacent to the collapse or destruction zone;

- calculation of heating plates and walls in the process of fire exposure.

As a result of the explosion, glazing and door fillings in apartments and on the staircase of the entrance were knocked out, several wall panels were deformed and knocked out, inter room partitions were destroyed. The dynamics of the increase in explosion pressure and maximum pressures on building constructions are reflected in [2]. A fire occurred on the 4th floor and the main influence of high temperature occurred on reinforced concrete prefabricated hollow core slabs between the 4th and 5th floors.

\section{Results and discussion}

As a result of the inspection, in the floor slabs between the 4th and 5th floors peeling of the protective layer of concrete from reinforcement and leaks during tapping was recorded, which indicates heating of reinforcement and concrete above a critical temperature and prolonged exposure to fire (confirmed by a calculated fire resistance limit, which is less than the fire exposure time) and the probable incompatibility of the work of concrete and reinforcing bar, the fact of incompatible operation of concrete and reinforcing bar was established visually), which could lead to the pulling (slipping) of the reinforcing bar and, as a result, to the collapse of the ceiling structure. Plate deflection [3] was recorded that exceeded the limit and reached its values as a result of a fire, which indirectly indicated the occurrence of plastic deformations in the reinforcing bar as a result of exposure to critical temperatures. A similar picture of a real fire was described in [4].

Rebar samples were taken from floor slabs, which were produced after analysis of defects and damage at the places of least damage to the load-bearing capacity of the slabs. In fig. 3 the results of tensile testing of reinforcing bars are presented, it is determined that both samples have a pronounced yield point in the neighbourhood of 550-570 MPa. 


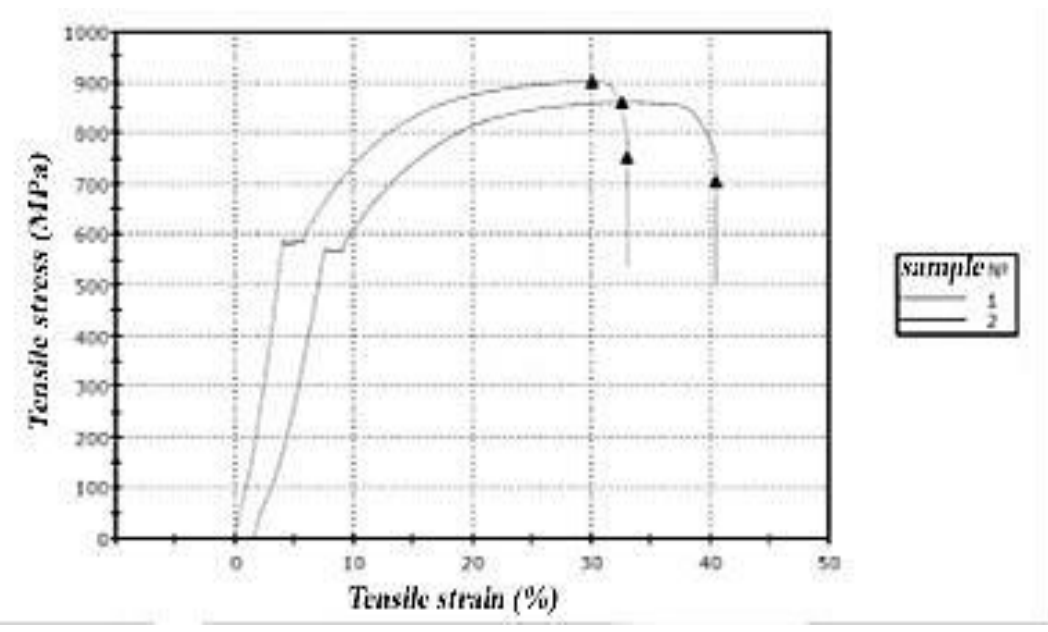

Fig. 3. Testing of the reinforcing bar. Sample 1 - Ø14 mm reinforcing bar; sample 2 - Ø12 mm reinforcing bar.

An analysis of the results showed that the reinforcing bar corresponds to class A-IIIв with a standard resistance of Rsn $=540 \mathrm{MPa}$. As a result of heat exposure, the strength characteristics of the reinforcing bar did not change. This issue was also considered in [58].

An instrumental examination determined the actual strength of concrete under compression in load-bearing wall panels and the overlap between the 4th and 5th floors of the building. It was found that the conditional class of concrete under compression (when testing cores taken from the indicated walls (fig. 4.) in load-bearing reinforced concrete panels of a building is from B10 to B20. The influence of temperature did not affect the strength characteristics of concrete in wall panels.

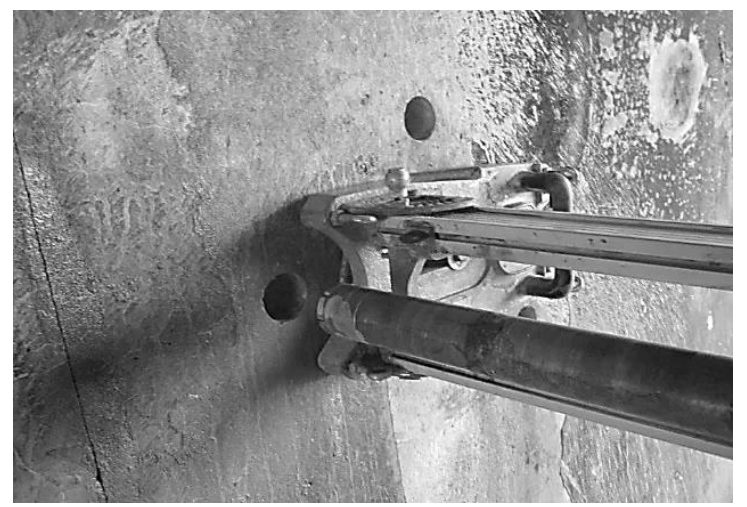

Fig. 4. Concrete sampling from the structure with a Hilti DD 130 diamond drilling rig.

For floor slabs, an additional ultrasound study of the strength of concrete was carried out. In some areas of floor slabs, a local decrease in concrete strength from B20 to B10 was recorded. This decrease was a result of an increase in the number of surface and internal defects (cracks and tears) in the concrete structure due to a sharp change in the temperature and humidity conditions when exposed to high temperature and water jet during extinguishing $[9,10]$. 
To determine the heating of reinforced concrete structures in real fires, we posed a mathematical problem to obtain a calculation equation characterizing the heating of an unlimited plate under fire exposure (see fig. 5).

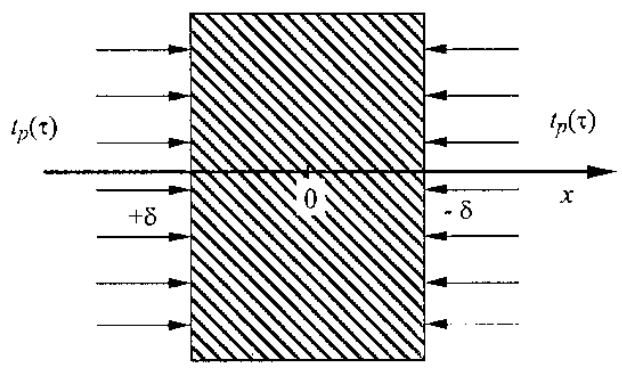

Fig. 5. Scheme of heating an unlimited plate.

To simplify the mathematical solution, we also assume that the temperature field of the plate at the initial moment and the heat transfer coefficient during the fire exposure are constant. As a result, the mathematical problem reduces to solving a system of equations

$$
\left\{\begin{array}{l}
\frac{\partial t}{\partial \tau}=a \frac{\partial^{2} t}{\partial x^{2}} \\
\left.t\right|_{\tau=0}=t_{0} \\
\left.\lambda \frac{\partial t}{\partial x}\right|_{x=0}=0 ;\left.\lambda \frac{\partial t}{\partial x}\right|_{x=\delta}-\left.\alpha[t p(\tau)-t(x, \tau)]\right|_{x=\delta}=0
\end{array}\right.
$$

The presence of the system of equations (1) in the second boundary condition that varies with time the fire temperature $t_{p}(\tau)$ significantly complicates the solution of this system of equations. However, having made the appropriate transformations, it was possible to obtain a solution based on the method of separation of Fourier variables [11] and analysis of research results of other authors [12-15].

Given the expression for $P_{n}(\tau)$, the solution to problem (1) takes the form

$$
t(x, \tau)=t_{p}(\tau)+W_{0} \sum_{n=1}^{\infty} A_{n} \cos \mu_{n} \frac{x}{\delta} e^{-\mu_{n}^{2}} \frac{a \tau}{\delta^{2}}+\sum_{n=1}^{\infty} A_{n} \cos \mu_{n} \frac{x}{\delta} \int_{0}^{\tau} e^{-\mu_{n}^{2} \frac{a}{\delta^{2}}(\tau-v)} f(v) \partial v
$$

Thus, we obtained the solution of the system of equations (1) in the form of an analytical formula (2) characterizing the heating of any point of an unbounded symmetrically heated plate under the influence of an arbitrarily changing temperature of a virtual fire.

Formula (2) allows one to obtain solutions for heating an unlimited plate with a known function of changing the temperature of a particular fire. For this in the place of $t_{p}(\tau)$ and $f(v)$ in formula (2) it is necessary to substitute the temperature functions corresponding to a particular fire.

An analysis of formula (2) shows that if at the initial time the plate temperature and fire temperature are equal, as is customary in calculating the fire resistance of building constructions, then in this case the value $W_{0}$ is zero and, therefore, in the place of (2) we get

$$
t(x, \tau)=t_{p}(\tau)+\sum_{n=1}^{\infty} A_{n} \cos \mu_{n} \frac{x}{\delta} \int_{0}^{\tau} f(v) e^{-\mu_{n}^{2} \frac{a}{\delta^{2}}(\tau-v)} \partial v
$$


On the other hand, if we assume that the temperature of the fire instantly takes the maximum value $t_{c}$, (which can occur during the combustion of flammable liquids and gases), then for this case the second sum in formula (3) will be zero, and we will obtain an analytical solution formulated problem, studied in detail in the theory of non-stationary heat conduction.

Warming up of reinforced concrete structures with high-intensity fire exposure.

To determine the degree of heating of reinforced concrete structures under conditions of high-intensity fire exposure, which can occur during the combustion of flammable liquids and gases, when the fire temperature at the initial time takes the maximum value, from the general solution (3), we obtain a calculation formula corresponding to the task.

It is known from the theory of unsteady heat conduction that the intensity of heating of solids largely depends not only on the temperature of the fire, but also on the conditions of heat transfer of the structure with a high-temperature gas medium, which is determined by the value of the Bio criterion $\left(B_{i}\right)$. Note that if the value of the criterion $B_{i}>50$, then the surface temperature of the solid during heating can be taken as the ambient temperature. This process is quite well known in the specialized literature and is widely used in computational practice. As for reinforced concrete structures, this case is characteristic of structural elements that are in direct contact with the flame at high values of the heat transfer coefficient. Thus, we come to the solution of the problem of unsteady heat conduction with boundary conditions of the first order. The solution for this case will be obtained from equation (3), which will take the form

$$
\frac{t_{c}-t(x, \tau)}{t_{c}-t_{0}}=\sum_{n=1}^{\infty} A_{n} \cos \mu_{n} \frac{x}{\delta} e^{-\mu_{n}^{2} \frac{a \tau}{\delta^{2}}}
$$

The obtained solution has been studied in sufficient detail in the theory of nonstationary heat conduction. To simplify the practical application, the right-hand side of this equation is presented in graphical form; therefore, the calculation of the heating of reinforced concrete structures for cases of extreme exposure to temperature conditions of real fires is performed according to the formula

$$
t(x, \tau)=t_{\tilde{n}}-\theta\left(t_{\tilde{n}}-t_{0}\right)
$$

where: $\theta$-relative excess temperature in the plate; $t_{c}$ - constant temperature of the fire flame, determined by calculation or by the results of a study of the temperature regime of a real fire.

The solution to the problem of heating an unlimited plate when the temperature of a real fire changes over time is proportional to the standard curve. In this case, we will consider some generalized version taking into account the temperature changes of real fires in proportion to the standard curve. The value of the coefficient of proportionality $\Psi$ may vary from 0.1 to 2.0 .

To obtain a decision on the temperature field in an unlimited plate heated by the temperature of the fire proportional to the standard curve, we assume that the temperature of the fire is determined by the equation

$$
t_{\Psi}(\tau)=t_{0}+\Psi \cdot 149,83 \ln (480 \tau+1)
$$

where: $\tau$ - in hours; $\Psi$ - proportionality coefficient, which may be greater or less than one.

Substituting equations (6) into (2), we obtain the desired solution in the form 


$$
\begin{aligned}
& t(x, \tau)=t_{0}+\Psi \cdot 149,83 \ln (480 \tau+1)- \\
& -\Psi \cdot 149,83 \sum_{n=1}^{\infty} A_{n} \cos \mu_{n} \frac{x}{\delta}\left\{\ln (480 \tau+1)+\sum_{m=1}^{\infty} \frac{\left(\mu_{n}^{2} \frac{a \tau}{\delta^{2}}\right)^{m}}{m \cdot m !}\right\} e^{-\mu_{n}^{2} \frac{a \tau}{\delta^{2}}}
\end{aligned}
$$

Converting to dimensionless variables and considering that $\ln \frac{1}{480}=6,17$, equation (7) can be written as

$$
\theta=\sum_{n=1}^{\infty} A_{n} \cos \mu_{n} \eta\left[1+\sum_{m=1}^{\infty} \frac{\left(\mu_{n}^{2} F_{0}\right)^{m}}{(\ln \tau+1,67) m \cdot m !}\right] \exp \left(-\mu_{n}^{2} F_{0}\right)
$$

where:

$$
\eta=\frac{x}{\delta} ; F_{0}=\frac{a \tau}{\delta^{2}} ; \theta=\frac{\left(t_{\Psi}(\tau)-t(x, \tau)\right)}{\left(t_{\Psi}(\tau)-t_{0}\right)}
$$

$t_{\Psi}(\tau)=t_{\text {пов }}(\tau)$ - real fire temperature proportional to the temperature of a standard fire.

Thus, for the first time, we obtained solution (8), which is important for practical calculations, which characterizes the heating of an unlimited plate under the influence of real fires, the temperature of which changes in proportion to the standard fire curve.

The following is a solution to the problem of heating a reinforced concrete floor slab when the temperature of the fire changes for the temperature conditions of a standard fire.

The calculation of the fire resistance of a reinforced concrete slab was made on the basis of loss of bearing capacity.

Initial data: section dimensions $b=1.6 \mathrm{~m}$; working span length $1=6.3 \mathrm{~m}$; section height $\mathrm{h}=220 \mathrm{~mm}$; the thickness of the protective layer of concrete to the bottom of the tensile reinforcing bar- $10 \mathrm{~mm}$; diameter of voids - $159 \mathrm{~mm}$. Heavy concrete, class B15, on silicate aggregate. Tensile reinforcing bar of class AIIIв Rsn $=540 \mathrm{MPa}$, Rs $=486 \mathrm{MPa} ; 9$ rods with a diameter of $14 \mathrm{~mm}$.

$$
\begin{aligned}
& \gamma_{s, T}=\frac{M}{h_{0} A_{s} R_{s u}} /\left(1-\frac{M}{2 b h_{0}^{2} R_{b u}}\right)=\frac{63500}{0,21 \cdot 13,86 \cdot 10^{-4} \cdot 486 \cdot 10^{6}} / \\
& \left(1-\frac{63500}{2 \cdot 1,6 \cdot 0.21^{2} 13,25 \cdot 10^{6}}\right)= \\
& =0,45 /(1-0,033)=0,45 / 0,967=0,47 \\
& t_{s}^{c r}=500+\frac{0,65-0,47}{0,65-0,45} \cdot 50=545^{0} C \\
& =\frac{1}{12 \alpha_{r e d}}\left(\frac{\delta_{s}+\varphi_{1} \sqrt{\alpha_{r e d}}+\varphi_{2} d_{s}}{\left.1-\sqrt{\frac{t_{s}^{c r}-20}{1200}}\right)^{2}=}\right. \\
& =\frac{1}{12 \cdot 0,001333}\left(\frac{0,01+0,62 \sqrt{0,001333}+0,5 \cdot 0,014}{1-\sqrt{\frac{545-20}{1200}}}\right)^{2}=0,88=R 52
\end{aligned}
$$


As a result of the calculations, it was found that the heating of the working reinforcing bar of the floor slab to a critical value is 52 minutes. Comparing the calculated value with the actual thermal exposure time of a real fire, which amounted to 80 minutes, we can conclude that the continued operation of floor slabs is impossible without taking measures to replace or strengthen them.

\section{Conclusion}

As a result of calculations and experimental studies, recommendations were issued on the restoration of a residential building after a gas explosion and subsequent fire. The exact scope of the restoration work was determined and a design solution was prepared for the restoration of the residential building.

Based on the recommendations issued, a set of repair and restoration works was carried out as soon as possible. In fig. 6 , a photograph of the house after restoration is shown.

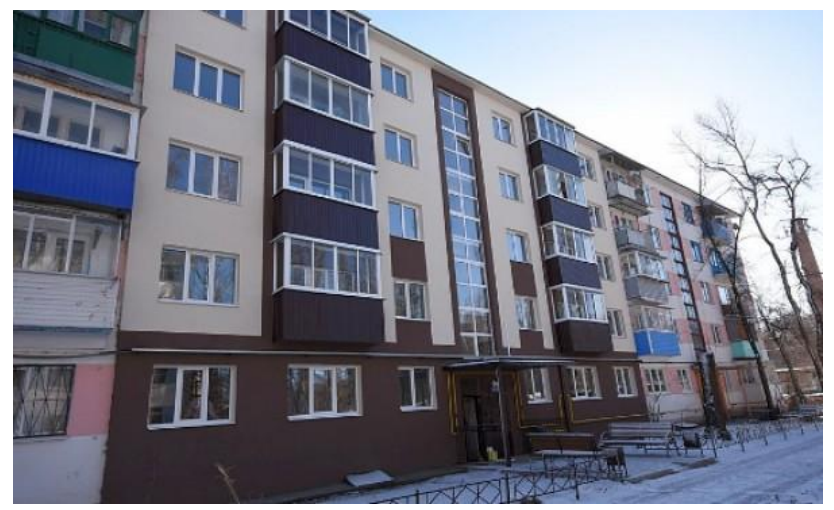

Fig. 6. Photo of the house after restoration.

The total cost of eliminating the consequences of the explosion and fire, as well as the restoration of a residential building amounted to 23.5 million rubles, the recovery area was 1100 square meters.

For comparison, the case of a similar explosion and subsequent fire in the city of Volgograd was studied as a result of which the entrance collapsed and a decision was made to demolish the building. The approximate indicators of damage only for the demolition of the building amounted to 16.3 million rubles. In addition to the cost of demolition, more than 250 million rubles were required. for the construction of a new house built on the site of demolition.

Thus, the results of the work done showed the possibility of restoring the building's load-bearing structures after the combined effects of the explosion and fire, as well as its effectiveness.

\section{References}

1. M. Arens, N.N. Brushlinsky, P. Wagner, S.V. Sokolov, Fire and explosion safety 10, 51-58 (2015)

2. K. Yang, Q. Hu, S. Sun, P. Lv, L. Pang, Journal of Loss Prevention in the Process Industries 62, 103969 (2019)

3. L. Lu, Y. Yuan, R. Caspeele, L. Taerwe, Fire Safety Journal 73, 29 (2015) 
4. P. Knyziak, R. Kowalski, J.R. Krentowski, Engineering Failure Analysis 97, 53-60 (2019).

5. EN 1992-1-2. Eurocode 2: Design of concrete structures - Part 1-2: General rules Structural fire design (2004).

6. R. Kowalski, Procedia Eng 193, 27-34 (2017)

7. M.Z. Naser, Automation in Construction 106, 102916 (2019)

8. Y. Wang, G. Yuan, Z. Huang, J. Lyu, B. Long, Fire Safety Journal 100, 171-185 (2018)

9. E. Ryu, H. Kim, Y. Chun, I. Yeo, Y. Shin, Engineering Structures 20715, 110165 (2020)

10. R. Kowalski, Struct. Concr. 8, 11-15 (2007)

11. A.M. Zaitsev, S. Gubskiy, Forestry technical magazine 4, 118-120 (2016)

12. R.T. Kuehnen, M.A. Youssef, Fire Safety Journal 108, 102831 (2019)

13. G.C. Thomas, A.H. Buchanan, C.M. Fleischmann, Fire Safety Science 5, 607-618 (1997)

14. M.A. Youssef, M. Moftah, Engineering Structures 29(10), 2618-2634 (2007)

15. J. Hwang, H. Kwak, Fire Safety Journal 100, 128-139 (2018) 